\title{
Cine y ética en contabilidad: una experiencia sobre la mejora en sensibilidad ética
}

Calabor, $\mathbf{M}^{\mathrm{a}}$, Merello, $\mathrm{P}^{\mathrm{b}}$ y Zorio, $\mathbf{A}^{\mathrm{c}}$

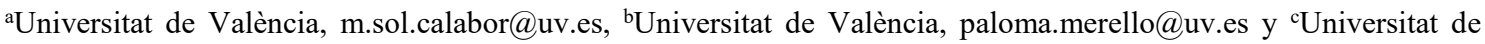
València, ana.zorio@uv.es

\section{\$EWWFW}

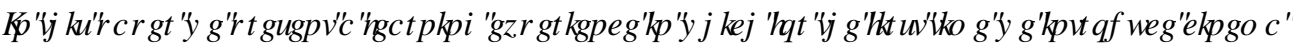

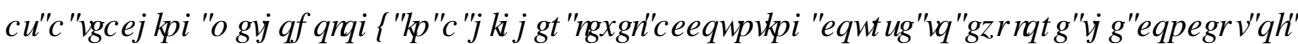
HKRIFDOMHQUИLWW

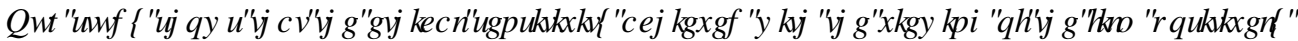

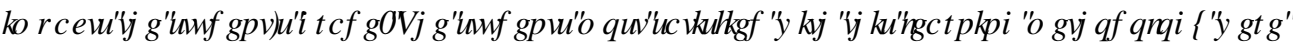

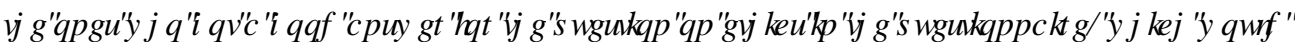

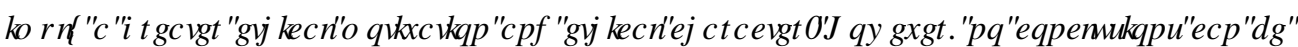
GDZ QDERXWWKHUJ QUIFDQFHRIIUKHIP SDFWRQ UKHTXDOIIFDMRQRIIHKIFDOP RUMDURQRRUUKH

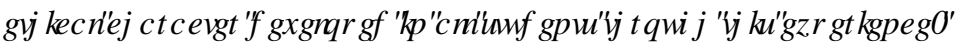

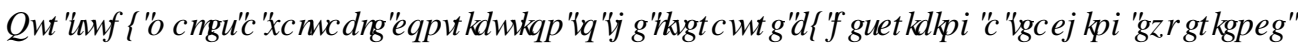

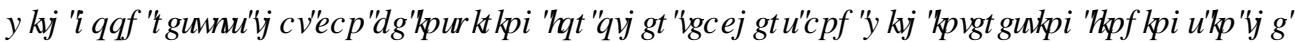

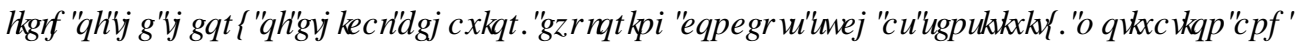

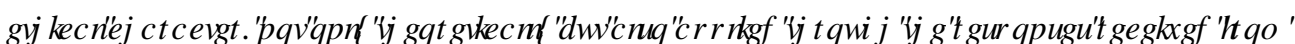

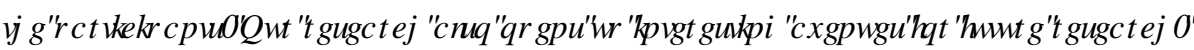

Keywords: film, accounting, ethics, Enron, education

प

\section{HXP HQ}

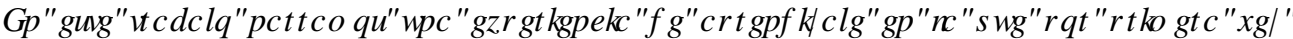

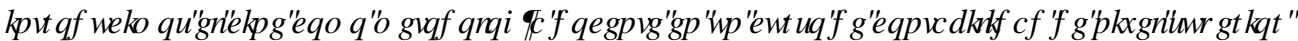

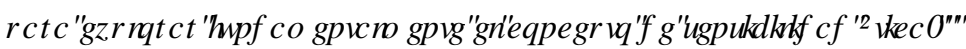

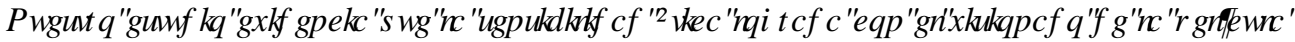

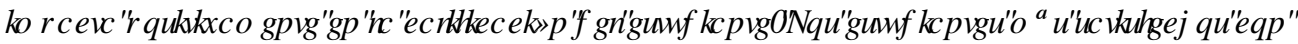

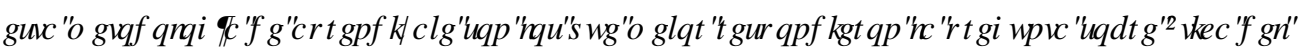

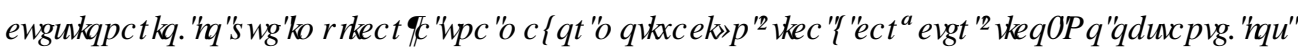

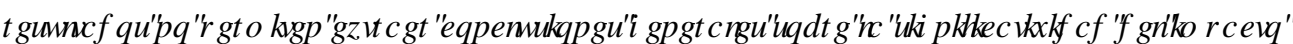

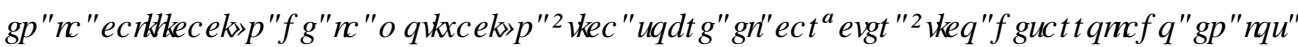

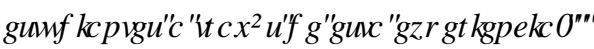

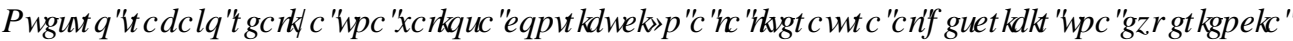

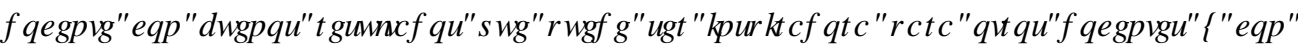

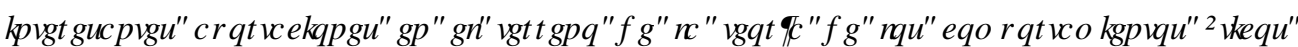

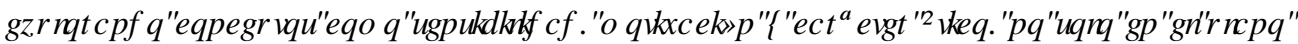




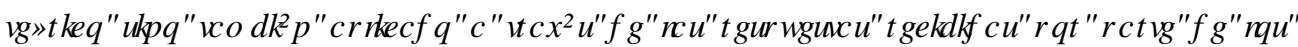

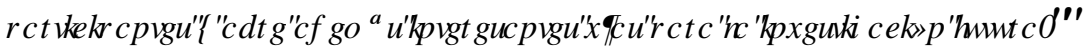

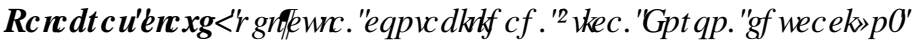

\section{Introducción}

La falta de ética se constituye como una de las causas de los numerosos escándalos financieros y contables producidos desde finales de los noventa. En este sentido, son múltiples los autores que afirman que la falta de ética y las prácticas fraudulentas apuntan a una dimensión moral de la crisis económica no suficientemente comprendida en todo su alcance (Ibrahim, Angelidis y Howard, 2006; Argandoña, 2009; Curtis, Harney y Jones, 2013; Fernández, 2014)

Haciendo referencia a titulaciones relacionadas con la gestión financiera y contable de las empresas, las instituciones universitarias han venido reflexionando sobre el papel que deben asumir en cuanto a la formación en valores éticos de sus estudiantes (Gonzalo y Garvey, 2007; Larrán y Andrade, 2015), con la finalidad no tanto de brindarles unos principios morales que ya deberían conforman su carácter sino en ayudarles a aplicarlos a su práctica profesional (Miller, Shawver y Mintz, 2020)

En general, la educación de la ética en contabilidad se ha centrado en el desarrollo del juicio ético del alumno partiendo de la premisa de que el juicio ético en contabilidad está rodeado de reglas y, por tanto, el conocimiento del sistema de reglas en todos los aspectos de la contabilidad (financiera, interna, auditoria y/o fiscal) apoyará su comportamiento ético (Uysal, 2010; Andersen y Klamm, 2018). Este enfoque pedagógico asume, por tanto, que hay una relación positiva entre el juicio ético y la toma de decisiones éticas. Sin embargo, la investigación no logra realmente evidenciar esta relación ya que son múltiples los factores que determinan una elección ética (por ejemplo, demográficos, edad, ideas políticas o nivel de estudios) y, por tanto, estudiar el juicio ético sin incluir la sensibilidad, la motivación y el carácter moral puede provocar esta falta de resultados concluyentes (Bailey, Scott, y Thoma, 2010; Christensen, Cote,y Latham, 2016). En este sentido, Liu, Yao y Hu (2012) discuten estas cuestiones en su trabajo sobre el enfoque de enseñanza de la ética en medicina y derecho y llegan a la conclusión de que la educación ética en contabilidad muestra deficiencas con respecto a estas disciplinas lo que produce estudiantes con menores capacidades cognitivas de tipo moral.

Por otra parte, el vínculo entre una conducta ética de los profesionales contables y su asociación a informes financieros de calidad es el fundamento sobre el que se construye la confianza en la actividad contable y en la información que ésta proporciona. Entendemos que la calidad de estos informes no sólo está sujeta al cumplimiento de la regulación, sino que presupone el cumplimiento de normas no escritas de comportamiento ético (Laballe, Gargouri, y Francouer, 2010; Giner y Pardo, 2015). Por ello y para mantener la confianza de los usuarios de la información, es importante que los profesionales de la contabilidad resuelvan e informen de los problemas éticos que encuentran en su actividad profesional (de motivación ética y carácter ético, como explicamos en el párrafo siguiente). En este sentido, la obligación moral de limitar el oportunismo rara vez se reconoce en la literatura financiera/contable hasta principios del presente siglo, cuando autores como Boatright (2007) sostienen que el funcionamiento de la economía requiere la práctica de las virtudes morales siendo los intermediarios, entre ellos los contables, los que tienen la responsabilidad de actuar como guardianes de estos valores.

Por tanto, si partimos del hecho de que los estudiantes tienen valores éticos inherentes, una educación eficaz en ética contable los ayudará a incorporar sus valores a los estándares éticos de la profesión (Sheehan y Schmidt, 2015; Stephenson, 2017; Andersen y Klamm, 2018). Este enfoque pedagógico implica ir más allá 
de la enseñanza de la ética como una cuestión de cumplimiento o de juicio ético, y se centra en la capacidad del estudiante para reconocer o discernir la presencia de problemas éticos (sensibilidad ética) en su capacidad para priorizar los valores éticos frente a otros valores (motivación ética) y la intención de tratar de resolver e informar sobre el problema ético detectado (carácter ético) (McPhail, 2004; Chan y Leung, 2006). La implicación que consideramos se desprende de este argumento es que, si la sensibilidad ética juega un papel significativo en las estructuras sociales y si es una parte inevitable de la toma de decisiones, seguramente merece más atención de la que se le ha prestado hasta la fecha en la educación contable y la ética contable.

Según Armstrong, Ketz, y Owsen (2003), la literatura relacionada con la educación ética en contabilidad revela que la mayoría de los autores realizan un razonamiento prescriptivo y por tanto los investigadores en contabilidad deberían tratar de explorar nuevas formas de mejorar la sensibilidad de los estudiantes de contabilidad. En este mismo sentido, autores como Massey y Van Hise (2009) o Christensen, Cote y Latham (2016) sugieren que diferentes pedagogías (por ejemplo, tareas reflexivas, análisis de casos o técnicas de aprendizaje activo) proporcionan un mayor impacto sobre el alumno en la enseñanza de la ética contable, sobre todo, si esta enseñanza se integra en el propio curso de contabilidad. Por tanto, las películas como recurso de instrucción en el aula permiten introducir un caso real que motive a los estudiantes a aprender captando su interés y agitando sus emociones (Bay y Felton, 2012). Desafiar a los estudiantes a pensar más allá de sus horizontes habituales presenta oportunidades de pensamiento y análisis crítico, ayuda a apreciar que el proceso de toma de decisiones éticas en el trabajo no es independiente, ni distinto de lo que se requiere en la vida cotidiana.

Una vez analizadas las deficiencias detectadas y la falta de consenso en cuanto a los resultados obtenidos en las investigaciones previas sobre la formación en valores éticos de los estudiantes de disciplinas contables, introducimos en este estudio el proceso cognitivo de sensibilidad ética. Específicamente, introducimos el cine como actividad de aprendizaje en un curso de contabilidad de nivel superior para explorar fundamentalmente el concepto de sensibilidad ética. Presentar a los estudiantes casos "paradigmáticos" que les muestren situaciones de conflicto relacionadas con los principios éticos en contabilidad, como el interés público, la independencia, la integridad o la objetividad, puede sin duda ayudarles a desarrollar su sensibilidad, motivación y carácter ético. Estos casos, junto con la posterior reflexión sobre los mismos, deberían permitir al alumno reconocer la presencia de problemas éticos y trasladar esa sensibilidad a su quehacer profesional, aplicando también una motivación ética y permitiéndoles actuar con carácter ético.

Tras esta breve introducción sobre la literatura previa en ética y educación contable, nuestro trabajo presenta los apartados de objetivos, descripción de la experiencia docente, resultados y conclusiones,

\section{Objetivos}

El objetivo de nuestro estudio es aportar evidencia de investigación sobre el uso de recursos filmográficos en un curso de contabilidad desde la perspectiva de los alumnos de Auditoria con el fin de arrojar luz sobre el papel que la sensibilidad, la motivación y el carácter ético deberían tener en la enseñanza de esta disciplina. Además, nuestro objetivo es explorar si a partir de la presentación de un caso como una secuencia de eventos que muestran el vínculo entre acciones y consecuencias, el alumno es capaz de cuestionar que determinados actos pueden no estar prohibidos expresamente en una norma o ley escrita, pero esto no implica que sea ético llevarlos a cabo. 
Lo que pretendemos en primera instancia es determinar si el alumno, sin indicaciones o reflexiones previas a la actividad por parte del profesor, sitúa el problema ético por encima de cualquier otra apreciación. Partimos de la presunción de que los alumnos ya tienen valores éticos en su vida personal y queremos saber si los extrapolan al mundo laboral- de forma que aquellos que seleccionen la opción contable del resumen que se explica a continuación estarán haciendo un juicio ético (no cumple la normativa), mientras que los que opten por el ético estarán aplicando su sensibilidad. Por eso, es interesante qué resumen eligen, en la medida que ello nos permite identificar si la importancia la sitúan en la ética, en la contabilidad o sencillamente no ven ninguna.

En cuanto al examen de la materia, cabe precisar que consta de dos partes: una parte teórica y otra de casos prácticos. La parte teórica es donde principalmente se evalúan los conceptos éticos. La cuestión sobre ética en la que se le plantea al alumno una situación concreta para que determine el incumplimiento o no de algún aspecto ético en el trabajo del auditor, en este caso la pregunta fue la siguiente:

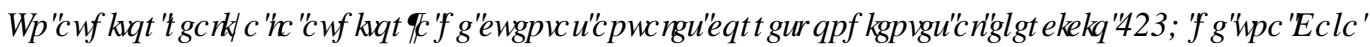

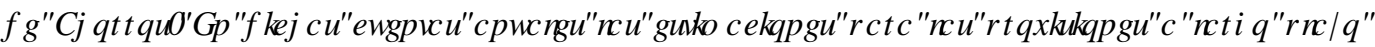

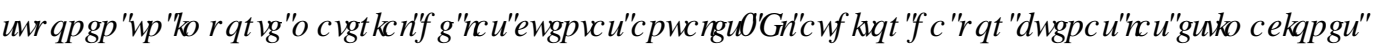

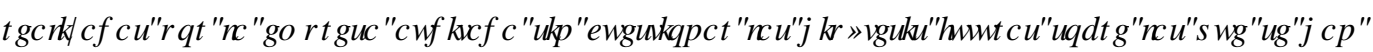

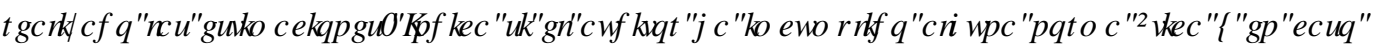

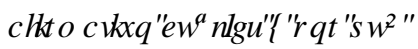

Los alumnos de la asignatura obtuvieron una media de 4,25 sobre 10 en la parte teórica del examen (desviación típica 2,56, mínimo 0 y máximo 8,75). En cuanto a la pregunta sobre ética, los estudiantes obtuvieron una nota media de 0,69 sobre 1 con una desviación estándar igual a 0,46.

Adicionalmente, y dado que la película facilitada es en versión inglesa y no ha sido doblada al español, nos proponemos analizar la competencia lingüística en este idioma de nuestros alumnos, a partir del grado de esfuerzo que realizan al visionar la película en versión original y su apreciación sobre si este esfuerzo les ayuda en la adquisición de esta competencia.

\section{Experiencia docente}

Las actividades de aprendizaje detalladas a continuación se incluyeron en la asignatura de Auditoria, impartida en el $4^{\circ}$ curso del Grado en Finanzas y Contabilidad de la Universitat de València, y se llevaron a cabo a lo largo del primer semestre del curso académico 2020/2021.

La muestra la conforman 66 alumnos matriculados en la asignatura. Aproximadamente el $66 \%$ de los alumnos están entre los 20 y los 24 años mientras que el 34\% restante se situan en edades superiores a los 24 años. Si nos centramos en los alumnos que siguieron la experiencia de evaluación continua, un total de 51 alumnos vieron la película y respondieron los cuestionarios, de los cuales 30 son mujeres y 21 hombres..

Para la elección de la película se siguieron los criterios de selección recomendados en investigaciones previas, en las que se aconseja que las películas reflejen situaciones plausibles, tengan cierto atractivo estético, se relacionen con los objetivos educativos del curso y, a ser posible, sean películas contemporáneas (Bay y Felton, 2012). Para maximizar la probabilidad de atraer el interés de los estudiantes, seleccionamos "The Crooked E - The Unshredded Truth About Enron"” (Spheeris, 2003). Basada en el libro de Brian

\footnotetext{
${ }^{1}$ https://www.youtube.com/watch?v=AiWKPQAWuug\& feature=youtu.be
} 
Cruver (2002) “Anatomy of Greed”, esta película para televisión narra el escándalo de Enron desde la perspectiva del autor (empleado entones en el gigante energético), se emitió por la cadena estadounidense CBS en enero de 2003 y supuso un éxito de audiencia. Elegimos esta película porque muestra cómo determinadas personas (en este caso, Jeffrey Skilling) son capaces de interpretar la ley para manipular a su favor los resquicios que esta deja, incumpliendo reglas éticas para obtener ventajas que les permitan ganar más dinero. Además, muestra a través de un caso real qué consecuencias pueden tener estas acciones para los individuos y para el conjunto de la sociedad.

La acción pedagógica consta de cuatro actividades diferenciadas que, de manera obligatoria, deben realizarse secuencialmente. Estas actividades, incluyendo el visionado de la película forman un conjunto y constituyen un $10 \%$ de la calificación total del curso si se realizan el total de actividades, por lo que la puntuación no tiene en ningún caso puntuaciones intermedias:

1. En primer lugar, se programó un cuestionario, a través del aula virtual de la asignatura, para recolectar los datos sociodemográficos de los alumnos participantes.

Dado que la película facilitada es en versión inglesa con la posibilidad de subtítulos en inglés nos pareció necesario conocer el nivel de idioma para comprobar que los participantes serían capaces de seguir el argumento, por lo que se les requirió también en el cuestionario su nivel de inglés.

2. La segunda actividad consistía en el visionado de la película fuera del horario de clase, para lo que se suministró el enlace correspondiente también desde el aula virtual, de manera que el sistema comprueba quién ha contestado al cuestionario inicial y, por tanto, ya puede realizar esta actividad, una vez realizada ésta le permite acceder a la siguiente.

Inicialmente se suministró información general sobre la repercusión que tuvieron los hechos que se relatan para la profesión de auditoria y cómo una de las cinco empresas más importantes del sector (las entonces denominadas $\% J \square$ ) $I Y H$, Arthur Andersen, fue condenada por obstrucción a la justicia y revocada su licencia para ejercer de auditora pública.

La intención era dejar la visualización inicial un tanto desestructurada, sin excesiva información o argumentación adicional, con el objetivo de fomentar una reflexión individual y una respuesta a los cuestionarios más espontánea. De esta manera, los estudiantes pueden practicar habilidades cognitivas de nivel superior (reflexión, metacognición o razonamiento, entre otras).

En ningún caso se impidió, pero tampoco se fomentó, que el alumno pudiera recurrir a otros recursos para obtener más información sobre el caso, para poder contrastar sus propias reflexiones o incluso para conocer las consecuencias reales de los sucesos detallados en el film. Hay que tener en cuenta que nuestra acción pedagógica no va dirigida a la instrucción sobre conceptos o a involucrar inicialmente al alumno en un juicio ético sobre el cumplimiento de normas. De hecho, es la única información que se facilitó: "Arthur Andersen incumplió la norma, y el sistema penal americano la condenó por ello"

Tras el visionado y el periodo reflexivo, se abrió un segundo cuestionario. En él se presentan al alumno tres resuménes de 250 palabras aproximadamente resaltando cada uno de ellos características de tipo filmográfico, contable o ético. Los tres resúmenes siguen la estructura de la sinopsis: introducción, nudo y desenlace, resaltando unos aspectos u otros de la película en función del tipo de resume, así:

- Resumen filmográfico: Se resalta la argumentación sin ningún tipo de connotación más allá del relato que hace el guionista.

- Resumen contable: En él se resaltan los aspectos contables que aparecen en el film como la contabilidad creativa, la ingeniería contable o la contabilidad de coberturas. Conceptos que aparecen implícitos y en algunos casos explícitos en la narrativa. 
- Resumen ético: En este resumen se resalta el incumplimiento de normas no escritas tal y como aparecen en la película y se apela a la responsabilidad ante determinadas actuaciones y a las consecuencias personales y sociales de esos actos.

El alumno escogerá un resumen de los tres anteriores, siendo el orden de aparición de los mismos dentro de la pregunta aleatorio y, por supuesto, sin respuesta correcta o incorrecta, ya que se le requiere su apreciación general- concretamente, a través de la pregunta siguiente:

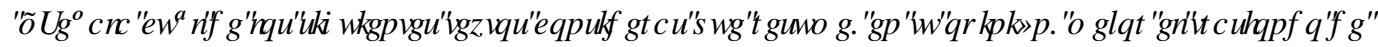
DOSHDFXD]"

En este mismo cuestionario se le plantean las siguientes preguntas con tres posibles respuestas:

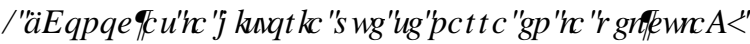

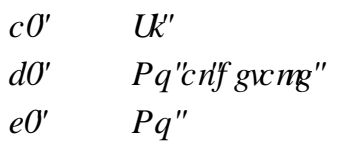

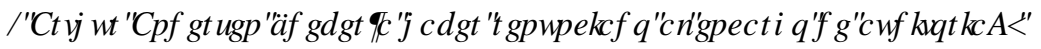

DD $\quad$ 6LISRUHMUUFRP SURP HMGDUXIQAHSHQCHQFWD

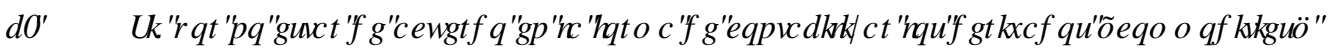

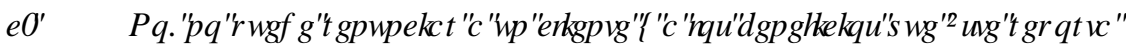

[

3. Por último, y también a partir de un cuestionario en aula virtual se les planteó la siguiente encuesta, con dos apartados diferenciados. En la tabla 1, se muestran las preguntas formuladas en el apartado de competencia lingüística. Por su parte, en la tabla 2 , se indican la preguntas formulada en cuanto a la metodología docente y valoradas en escala Likert de 5 puntos (del 1 Totalmente en desacuerdo al 5 Totalmente de acuerdo)

$7 D E D \square \square \& R P S H A P W Q Q D \square T W A D$

\begin{tabular}{|c|c|}
\hline Pregunta & Opciones de respuesta \\
\hline \multirow{4}{*}{$\begin{array}{l}\text { ¿Habitualmente ves películas de habla inglesa en } \\
\text { versión original? }\end{array}$} & Sin subtítulos \\
\hline & Con subtítulos en inglés \\
\hline & Con subtítulos en español \\
\hline & Siempre dobladas al español \\
\hline \multirow{3}{*}{ ¿Cómo has visto la película? } & Sin subtítulos \\
\hline & Con subtítulos en inglés \\
\hline & Con subtítulos en español \\
\hline \multirow{2}{*}{$\begin{array}{l}\text { ¿Te ha sorprendido, en positivo, tu nivel de } \\
\text { comprensión del idioma? }\end{array}$} & $\mathrm{Si}$ \\
\hline & No \\
\hline
\end{tabular}


7DEQDI [O HRRRRJTDGRFHUI

En una escala del 1 al 5, dónde 1 es "Totalmente en desacuerdo" y 5 "Totalmente de acuerdo". Valore las siguientes afirmaciones sobre la película:

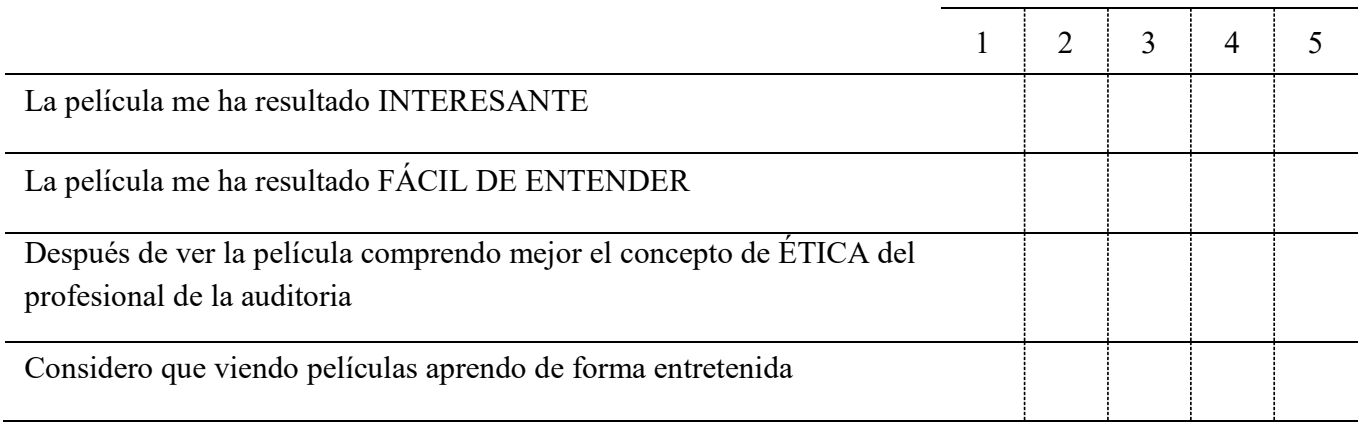

\section{Resultados}

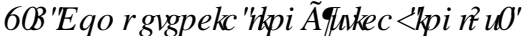

En cuanto a la competencia lingüística en lengua inglesa, el plan de estudios de la titulación incita a introducir y aumentar las competencias en inglés de los estudiantes. En línea con esto, analizamos cómo los estudiantes afrontan la tarea de ver una película en una lengua no materna y si este esfuerzo les ayuda en la adquisición de dicha competencia lingüística.

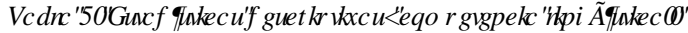

\begin{tabular}{|l|cccc|}
\cline { 2 - 5 } \multicolumn{1}{c|}{} & \multicolumn{4}{c|}{ ¿Cómo ves normalmente las películas rodadas en inglés? } \\
\hline $\begin{array}{l}\text { ¿Cómo has visto la película } \\
\text { del caso Enron? }\end{array}$ & En inglés & Subtitulos en inglés & Subtitulos en castellano & En castellano \\
\hline Inglés & $100 \%$ & $0 \%$ & $0 \%$ & $4 \%$ \\
\hline Con subtitulos en inglés & $0 \%$ & $82 \%$ & $36 \%$ & $35 \%$ \\
\hline Con subtitulos en castellano & $0 \%$ & $18 \%$ & $64 \%$ & $61 \%$ \\
\hline Total & $100 \%$ & $100 \%$ & $100 \%$ & $100 \%$ \\
\hline
\end{tabular}

La Tabla 3 evidencia que los estudiantes seleccionaron el idioma o subtítulos de la película de acuerdo a sus habilidades y para sentirse cómodos. De esta forma, el $82 \%$ de los estudiantes que normalmente ven las películas en inglés con subtítulos en inglés han visto el caso Enron siguiendo el mismo criterio. Solo un $35 \%$ de los estudiantes que normalmente ven las películas en español o con subtítulos en español se han esforzado por ver el caso Enron con subtítulos en inglés. Definimos a esos estudiantes como aquellos que se han esforzado por adquirir la competencia lingüística (grupo "esfuerzo").

Después de ver el caso Enron, preguntamos a los estudiantes si se han sorprendido de su comprensión del idioma. La tabla 4 muestra el porcentaje de alumnos según si pertenecen al grupo que se ha esforzado con el inglés y adquisición de la competencia lingüística, entendidendo esta última como percibir una mejora en el nivel de conocimiento de dicha lengua. 


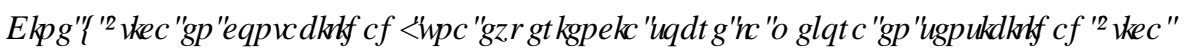

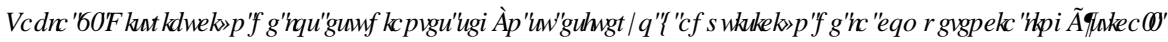

Sorpresa sobre la comprensión del idioma

\begin{tabular}{llll}
\hline Esfuerzo & NO & SI & Total \\
\hline NO & $75 \%$ & $25 \%$ & $100 \%$ \\
\hline SI & $23 \%$ & $77 \%$ & $100 \%$ \\
\hline
\end{tabular}

Como muestra la Tabla 4, el 77\% de los estudiantes que se esforzaron por ver el caso Enron con subtítulos en inglés (grupo "esfuerzo") se sorprendieron por su comprensión de la película. Además, el 75\% de los que se encontraban dentro de su zona de confort no se sorprendieron por sus habilidades lingüísticas. Esto sugiere que quienes usan subtítulos en español no se esfuerzan por escuchar ni comprender el idioma inglés y quienes están acostumbrados a ver películas en inglés sin subtítulos tampoco se sorprenden, como cabía esperar. Así pues, los resultados evidencian que incorporar tareas en un idioma extranjero y promover el esfuerzo sacando a los estudiantes de su zona de confort contribuye a la adquisición de estas habilidades, por lo que como sugerencia a futuro sería recomendable incorporar más tareas voluntarias en idioma extranjero y con subtítulos preferiblemente solo en inglés, para favorecer esta adquisión de competencias del grado.

Además, tratamos de comprobar si existe alguna diferencia en la selección del resumen ético o contable para aquellos alumnos que han hecho un esfuerzo por mejorar su competencia lingüística. Sin embargo, la tabla 5 no evidencia diferencias en la selección de los resúmenes. Esto sugiere que el esfuerzo en el idioma no nativo no se correlaciona con la preferencia por destacar los conceptos contables o éticos en el resumen que elige el estudiante como más representativo de la película.

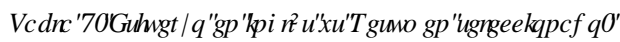

Esfuerzo con inglés

\begin{tabular}{|c|c|c|c|c|}
\hline & \multicolumn{2}{|c|}{ observaciones } & \multicolumn{2}{|c|}{ porcentaje } \\
\hline & no & sí & no & sí \\
\hline \multicolumn{5}{|l|}{ Resumen } \\
\hline filmográfico & 11 & 3 & $29 \%$ & $23 \%$ \\
\hline contable & 2 & 3 & $5 \%$ & $23 \%$ \\
\hline ético & 25 & 7 & $66 \%$ & $54 \%$ \\
\hline TOTAL & 38 & 13 & $100 \%$ & $100 \%$ \\
\hline
\end{tabular}

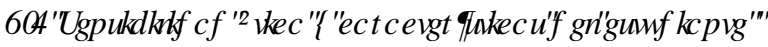

A continuación se detallan las características socio-demográficas principales de los estudiantes en función del resumen escogido. Como podemos comprobar, el 63\% de los estudiantes dispone de sensibilidad ética y escoge el resumen ético como aquel que mejor describe los rasgos más importantes de la película.

En la tabla 6, apreciamos las diferencias de selección del reusmen en función de las características sociodemográficas del estudiante. 


\begin{tabular}{|c|c|c|c|c|c|c|c|c|}
\hline & \multicolumn{4}{|c|}{ Resumen } & \multicolumn{3}{|c|}{ Resumen } \\
\hline & & filmográfico & contable & ético & TOTAL & filmográfico & contable & ético \\
\hline \multicolumn{9}{|l|}{ Género } \\
\hline & mujer & 8 & 3 & 19 & 30 & $27 \%$ & $10 \%$ & $63 \%$ \\
\hline & hombre & 6 & 2 & 13 & 21 & $29 \%$ & $10 \%$ & $62 \%$ \\
\hline \multicolumn{9}{|c|}{ Estudios de los padres } \\
\hline & primarios & 2 & 0 & 7 & 9 & $22 \%$ & $0 \%$ & $78 \%$ \\
\hline & secundarios & 9 & 0 & 7 & 16 & $56 \%$ & $0 \%$ & $44 \%$ \\
\hline & $\begin{array}{l}\text { bachillerato } \\
\text { universitario }\end{array}$ & 0 & 3 & 10 & 13 & $0 \%$ & $23 \%$ & $77 \%$ \\
\hline & $\mathrm{s}$ & 3 & 2 & 8 & 13 & $23 \%$ & $15 \%$ & $62 \%$ \\
\hline \multicolumn{9}{|c|}{ Nota acceso universidad } \\
\hline & $<8,5$ & 2 & 2 & 10 & 14 & $14 \%$ & $14 \%$ & $71 \%$ \\
\hline & $8,5-10$ & 4 & 2 & 14 & 20 & $20 \%$ & $10 \%$ & $70 \%$ \\
\hline & $10-12$ & 8 & 1 & 8 & 17 & $47 \%$ & $6 \%$ & $47 \%$ \\
\hline \multicolumn{9}{|l|}{ Ocupación } \\
\hline & $\begin{array}{l}\text { estudia } \\
\text { estudia y }\end{array}$ & 10 & 3 & 23 & 36 & $28 \%$ & $8 \%$ & $64 \%$ \\
\hline & trabaja & 4 & 2 & 9 & 15 & $27 \%$ & $13 \%$ & $60 \%$ \\
\hline \multicolumn{9}{|c|}{ Conocimiento previo del caso Enron } \\
\hline & no & 1 & 1 & 1 & 3 & $33 \%$ & $33 \%$ & $33 \%$ \\
\hline & no al detalle & 5 & 0 & 14 & 19 & $26 \%$ & $0 \%$ & $74 \%$ \\
\hline & si & 8 & 4 & 16 & 28 & $29 \%$ & $14 \%$ & $57 \%$ \\
\hline & TOTAL & 14 & 5 & 32 & 51 & $27 \%$ & $10 \%$ & $63 \%$ \\
\hline
\end{tabular}

Como podemos apreciar en la tabla 6 , no existen diferencias de género en la sensibilidad ética ya que tanto el $63 \%$ como el $62 \%$ de las mujeres y hombres, respectivamente, se decantan por este resumen.

El nivel cultural de los estudiantes pone de manifiesto una de las principales diferencias. Los alumnos cuyos padres tienen estudios secundarios priorizan los aspectos filmográficos frente a aquellos con padres con estudios primarios o bachillerato que destacan por su elevada sensibilidad. Por último, solo el $47 \%$ de los alumnos con mayor nota de acceso a la universidad seleccionan el resumen ético.

De otro lado, el conocimiento previo sobre el caso Enron parece que tiene una cierta influencia y predispone hacia la sensibilidad.

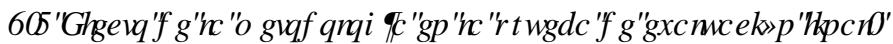

Para evaluar si la metodología propuesta tiene un impacto positivo en la adquisición final de los principales conceptos de la asignatura, desarrollamos diferentes análisis.

En primer lugar, analizamos si la respuesta a la pregunta del comportamiento de Andersen en el test del caso Enron y la sensibilidad del estudiante (selección de la respuesta "a"), muestra una influencia en las respuestas de la parte teórica del examen. 
La tabla 7 evidencia que existen diferencias significativas en la nota obtenida en la parte de teoría del examen entre aquellos alumnos que evidencian sensibilidad ética (selección de la opción a) y los que evidencian juicio normativo-contable (respuesta b), obteniendo una mejor calificación los primeros en la teoría del examen.

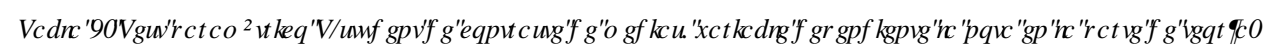

\begin{tabular}{ccccc} 
Respuesta a pregunta ética Andersen & Media & Error estándar & T Student & p-valor \\
\hline a & 5.945 & 0.186 & & \\
b & 5.250 & 0.311 & & \\
\hline & & & 2.039 & $0.024^{* *}$ \\
\hline
\end{tabular}

De otro lado, realizamos un análisis de correspondencias donde se relacionan dos variables cualitativas y se proporciona un mapa de semejanzas. En primer lugar, evaluamos si el resumen seleccionado está relacionado con la nota final obtenida en el examen. La figura 1 muestra que quienes seleccionaron el resumen filmográfico o contable obtienen una calificación final más baja en el examen, mientras que quienes obtienen un notable son los que identificaron el resumen ético.

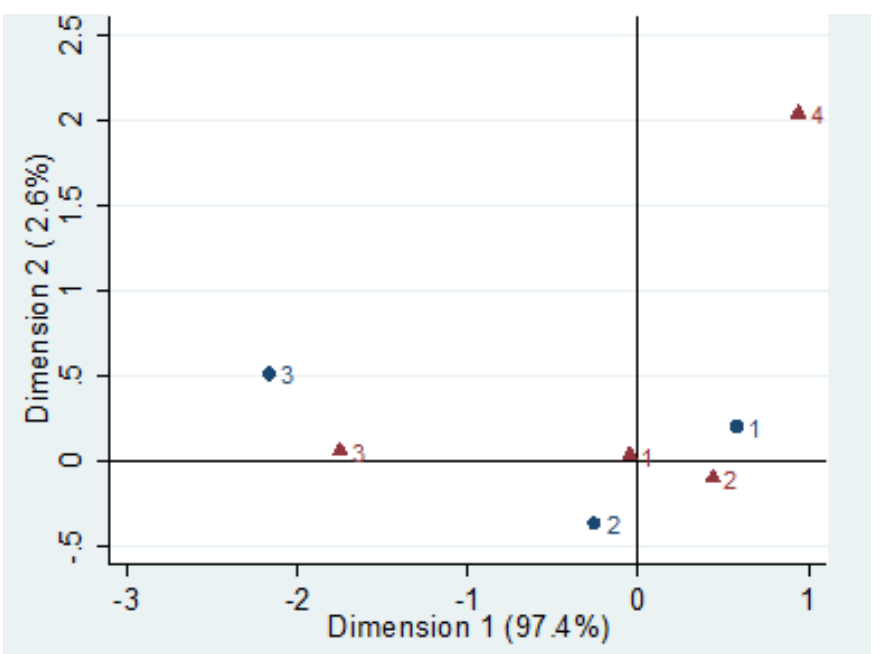

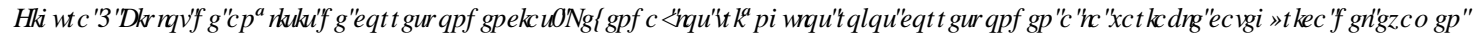

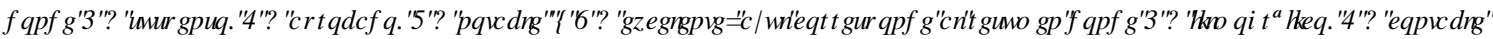
Iए। एpWFR

$\square$

Además, queremos analizar si seguir la metodología favorece la adquisición de los conceptos éticos.

Por un lado, evaluamos si ver la película tiene un impacto positivo en la nota final, independientemente de la inclinación ética del alumno en las respuestas en el caso Enron. En línea con esto, seleccionamos las preguntas teóricas del examen porque son las que registran el núcleo de los conceptos éticos. Realizamos una prueba no paramétrica (U-Mann Whitney) para evaluar las diferencias en la nota teórica del examen entre quienes siguen la metodología propuesta y quienes no. El resultado de la prueba es significativo ( $\mathrm{z}=$ -4.224, p-value $<0.01$ ), lo que indica que rechazamos la hipótesis nula y existe evidencia de que existe una diferencia en la puntuación de la parte teórica del examen para quienes han visto la película y los que no, obteniendo en promedio una nota superior a los que han participado en la metodología propuesta.

Sin embargo, realizamos una segunda prueba para ver si aquellos que respondieron correctamente la pregunta de ética del caso Enron obtuvieron calificaciones significativamente diferentes en la parte teórica 
del examen. En este caso el p-value $=0,849$, por lo que no podemos rechazar la hipótesis nula y por tanto afirmamos que seleccionar correctamente el comportamiento ético de Andersen en el caso Enron no garantiza necesariamente una mejora en la calificación, donde solo afecta el visionado. A la luz de estos resultados, parece que los estudiantes que participan en la actividad generan una sensibilidad ética con impacto en la calificación, pero en general hay campo de mejora en términos de motivación ética y carácter ético.

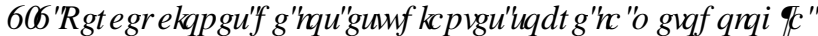

Como ya se ha comentado, los estudiantes responden a un cuestionario sobre su percepción de la película y la metodología. De esta manera se consideran cuatro variables cuantitativas tomando para ellos los valores de la escala Likert de 1 a 5 , siendo 5 totalmente de acuerdo. Las preguntas son: (i) creo que la película es interesante, (ii) creo que la película es fácil de entender, (iii) creo que la película me ha ayudado a comprender un concepto contable o financiero y (iv) considero que viendo películas aprendo de una manera entretenida.

Para analizar las percepciones de los estudiantes realizamos un análisis clúster de $\mathrm{k}$ medias, utilizando la distancia euclidiana y considerando las cinco preguntas del cuestionario de metodología. La inspección gráfica previa de las biplots de las cuatro variables evidenció la existencia de tres grupos particulares de estudiantes.

La Tabla 8 muestra los centros de los tres clusters así como la proporción de estudiantes que componen los grupos.

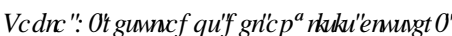

\begin{tabular}{rccccc} 
cluster & interesante & fácil & ayuda & aprendo & $\%$ obs \\
\hline 1 & 4.36 & 3.42 & 4.54 & 4.33 & $67 \%$ \\
2 & 3.00 & 2.75 & 3.25 & 4.75 & $16 \%$ \\
3 & 4.50 & 2.50 & 2.50 & 3.75 & $16 \%$ \\
\hline
\end{tabular}

El análisis de la Tabla 8 sugiere los siguientes perfiles:

- Grupo 1: está compuesto por los estudiantes más satisfechos que encuentran la película interesante, fácil y útil.

- Cluster 2: está compuesto por aquellos estudiantes que, estando muy satisfechos con la metodología, perciben la película como difícil y no muy interesante.

- Cluster 3: está formado por aquellos estudiantes que encuentran más difícil la película y, en consecuencia, no entendieron mejor el concepto ético.

Realizamos análisis de correspondencia para comparar si el perfil de los estudiantes se relaciona con los resultados del examen (parte teórica del examen donde se discuten los conceptos éticos). 


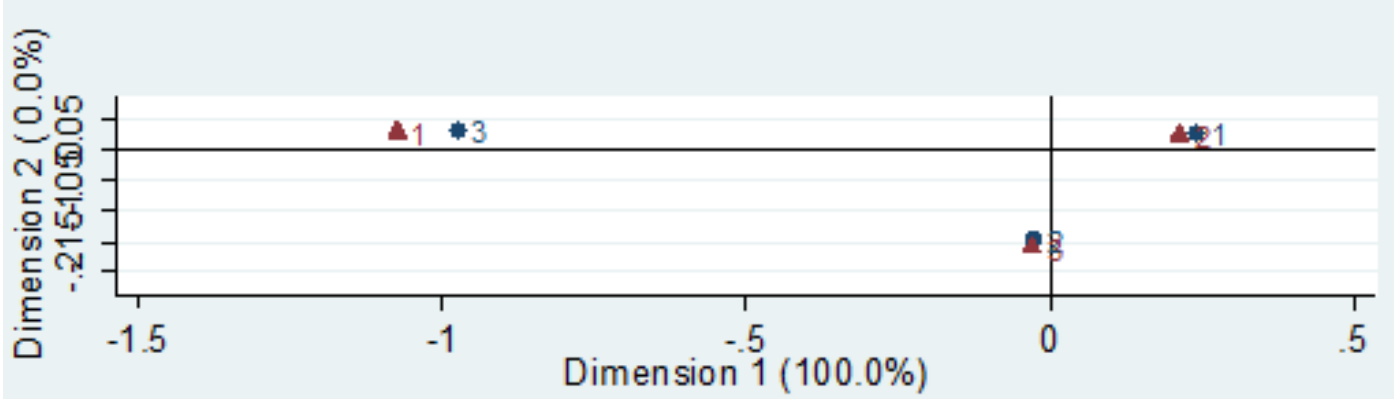

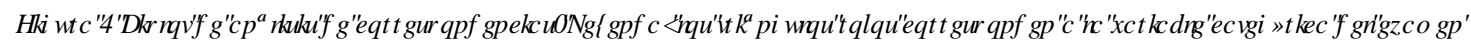

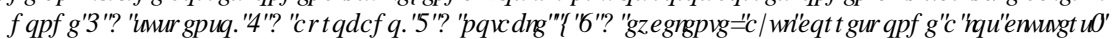

Como podemos ver en la Figura 2, aquellos estudiantes que no encontraron interesante la película (cluster 3) no aprobaron las preguntas teóricas (triangulo 1). Por otro lado, aquellos alumnos más satisfechos con la metodología (cluster 1) parecen obtener una nota de aprobado en la parte teórica del examen y los que menos les gustó la película (cluster 2) se relacionan más con una buena nota (notable).

Si seguimos el mismo procedimiento para la pregunta ética en el examen conseguimos que los estudiantes más satisfechos con la metodología (grupo 1) se relacionen con una respuesta correcta de la pregunta ética en el examen, mientras que aquellos que percibieron difícil la película (grupo 3) se relacionan con una respuesta incorrecta.

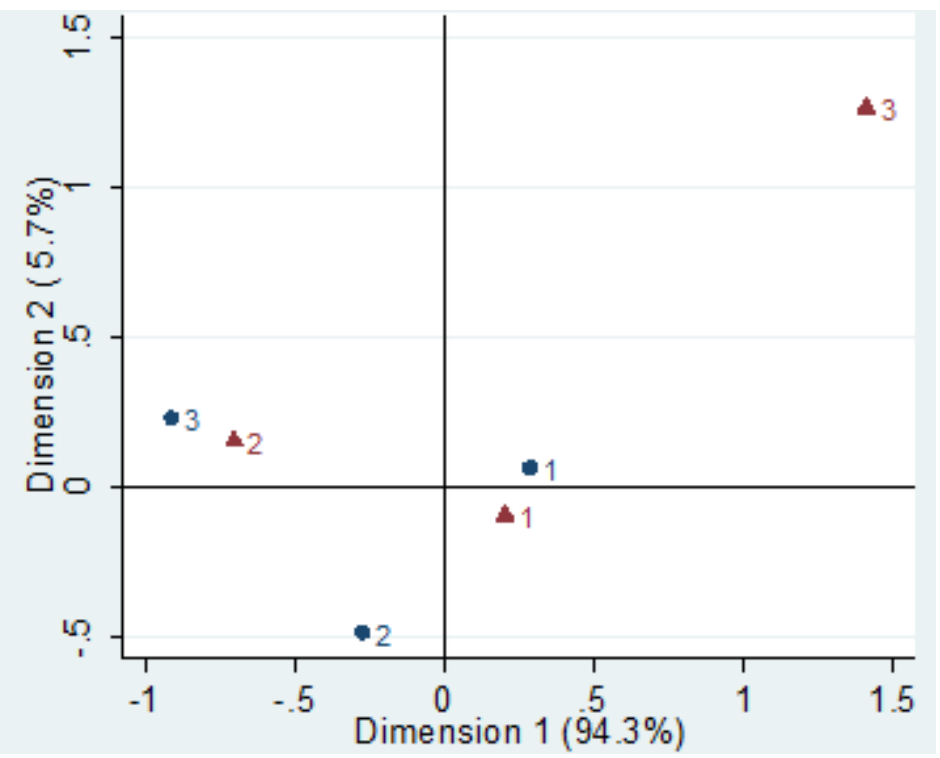




\section{Conclusiones}

Este trabajo presenta una experiencia innovadora de utilización del visionado de una película para introducir a los estudiantes de último curso de grado en la importancia de la ética en el comportamiento de los contables.

El estudio presenta una revisión de la literatura que incide en la importancia de promover los conceptos de sensibilidad ética, la motivación ética y el carácter ético. A través de un cuestionario, se recogen las respuestas de los estudiantes que han visionado una película sobre el caso Enron, de importantes implicaciones para la profesión contable dada su trascendencia no solo con la desaparición de una importante firma de auditoría a nivel global (Arthur Andersen), sino también por sus impactos posteriores en la regulación. Estas respuestas son analizadas en nuestro trabajo para extraer importantes conclusiones.

En primer lugar, cabe destacar que nuestro estudio evidencia que la sensibilidad ética lograda con el visionado de la película impacta positivamente en la calificación del estudiante. No obstante, nuestro estudio no permite extraer conclusiones sobre la significatividad del impacto en la calificación de la motivación ética o del carácter ético desarrollado en los estudiantes a través de esta experiencia. Curiosamente, también observamos que los estudiantes más satisfechos con esta metodología de aprendizaje son los que mejor respondieron la pregunta sobre ética del cuestionario, lo que implicaría una mayor motivación ética y carácter ético. No obstante, este grupo de estudiantes tienden a sacar una nota de aprobado en la nota de la parte teórica del examen, mientras que los menos satisfechos con la metodología sacan notable.

Respecto a la cuestión del idioma, se aprecia que hay una proporción de estudiantes que realiza un esfuerzo voluntario para la adquisición de la competencia lingüística visionando la película sin subtitulos en castellano y que tras este esfuerzo dichos estudiantes evidencian sorpresa al comprender el idioma mejor de lo que esperaban.

Nuestro trabajo realiza una valiosa contribución a la literatura en la medida en que se describe una experiencia docente con buenos resultados que puede ser inspiradora para otros docentes y con interesantes aportaciones en el terreno de la teoría de los comportamientos éticos explorando conceptos como sensibilidad, motivación y carácter ético, no solo en el plano teórico sino también aplicado a través de las respuestas recibidas por parte de los participantes.

Nuestra experiencia, abre caminos para la investigación futura pues sería muy interesante en los cursos académicos siguientes introducir nuevas películas a lo largo del semestre y plantear una batería de preguntas más amplia para medir con más detalle si hay mejoras en sensibilidad, motivación y carácter ético de los estudiantes a medida que van viendo más películas y que han reflexionado ya en el contexto de una película previa sobre toda la problemática de los planteamientos éticos. 


\section{Referencias}

Andersen, M. L., \& Klamm, B. K. (2018). Haidt's social intuitionist model: What are the

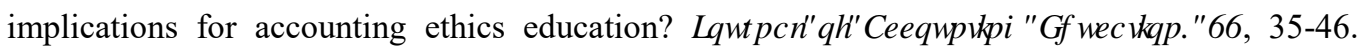
doi:10.1016/j.jaccedu.2018.05.001

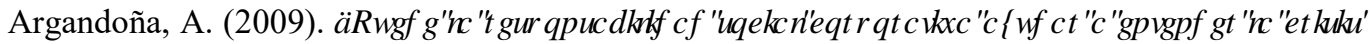
IIQDQFH $D^{\prime}$ IESE Business Schoool-Universidad de Navarra. Cátedra "la Caixa" de Responsabilidad Social de la Empresa y Gobierno Corporativo.

Armstrong, M. B., Ketz, J. E., \& Owsen, D. (2003). Ethics education in accounting: moving

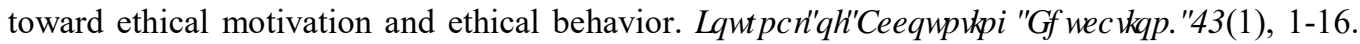
doi:10.1016/S0748-5751(02)00017-9

Bailey, C. D., Scott, I., \& Thoma, S. J. (2010). Revitalizing accounting ethics research in the neoKohlbergian framework: Putting the DIT into perspective. \%KDVIRLO5 HMHFKILQ $\$$ FFRXQUQJW पर(2), 1-26. doi:10.2308/bria.2010.22.2.1

Bay, D., \& Felton, S. (2012). Using Popular Film As A Teaching Resource In Accounting Classes. \$P HUFDQ-RXLQDOR II\%XUQHML( GXFDMRQW (2), 159-.

Boatright, J. R. (2007). Reluctant Guardians: The Moral Responsibility of Gatekeepers. \%XUQHM ( WKIFVA XDUИНФாए(4), 613-632. doi:10.5840/beq20071742

Chan , S. Y., \& Leung, P. (2006). The effects of accounting students' ethical reasoning and

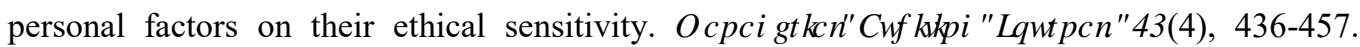
doi:10.1108/02686900610661432

Christensen, A. L., Cote, J., \& Latham, C. K. (2016). Insights Regarding the Applicability of the Defining Issues Test to Advance Ethics Research with Accounting Students: A Meta-analytic

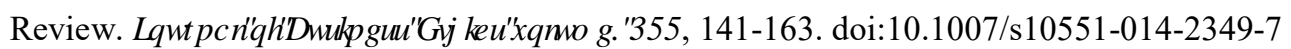

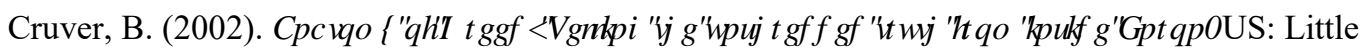
Brown.

Curtis, R., Harney, S., \& Jones, C. (2013). Ethics in a time of crisis: editorial introduction to special focus. \%XVQHML( WKIFVDC( XLRSHDQ5 HIIHUW (1), 64-67. doi:10.1111/beer.12008

Fernández, J. L. (2014). La falta de ética como concausa de la crisis económica. \%RQHQQ $G H$ ( WXGRV ( FRQ P IFRV QT(211), 25-42. Available at https://search.proquest.com/scholarlyjournals/la-falta-de-ética-como-concausa-crisis-económica/docview/1537380713/se-

2? accountid $=14777$

Giner, B., \& Pardo, F. (2015). How Ethical are Managers' Goodwill Impairment Decisions. - RХLQDORП\%ХИQHМ( UKIFVID०, 21-40. doi:10.1007/s10551-014-2303-8

Gonzalo, J. A., \& Garvey, A. M. (2007). Ética y enseñanza de la Contabilidad (Una propuesta de discusión). \&RQUDGXUD8 QIYHUGDCEGH\$ QURTXtDID, 11-42.

Ibrahim, N., Angelidis, J., \& Howard, D. (2006). Corporate social responsibility: A comparative analysis of perceptions of practicing accountants and accounting students. - RXLQDORIロ\%ХQQHW ( UसIFل⿴囗十 (2/3), 157-167. doi:10.1007/s10551-005-5572-4

Laballe, R., Gargouri, R. M., \& Francouer, C. (2010). Ethics, diversity management, and financial

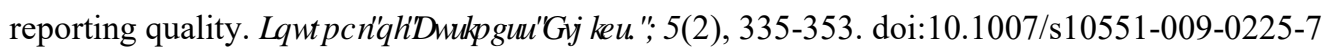


Larrán, M., \& Andrade, F. J. (2015). La oferta de asignaturas de responsabilidad social corporativa y ética empresarial en las titulaciones de finanzas y contabilidad: análisis comparativo con el

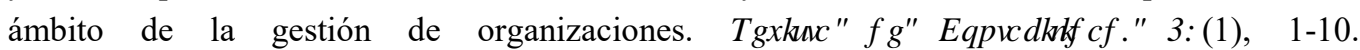
doi:10.1016/j.rcsar.2013.10.001

Liu, C., Yao, L. J., \& Hu, H. (2012). Improving ethics education in accounting: Lessons from medicine and law. , WXHLIQ\$ FFRXQMQJL( GXFDURQس (3), 671-690. doi:10.2308/iace-50150

Massey, D. W., \& Van Hise, J. (2009). Walking the walk: Integrating lessons from multiple perspectives in the development of an accounting ethics course. ,WXHIIQ\$ FFRXQMQJ L( GXFDURQL पि(4), 484-510. doi:10.2308/iace.2009.24.4.481

McPhail, K. (2004). An emotional response to the state of accounting education: developing accounting students' emotional intelligence. \&UAFDO3 HUSHFWHWLQ \$ FFRXQMQJWU(4-5), 629648. doi:10.1016/S1045-2354(03)00050-9

Miller, W. F., Shawver, T. J., \& Mintz, S. M. (2020). Measuring the value of integrating GVV

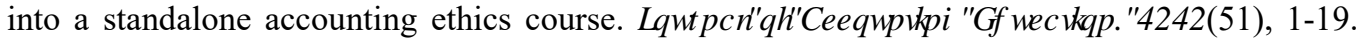
doi:10.1016/j.jaccedu.2020.100669

Sheehan, N. T., \& Schmidt, J. A. (2015). Preparing accounting students for ethical decision making: Developing individual codes of conduct based on personal values. - RXLQDORI\$ FFRXQMQJU ( GXFDMRQM (3), 183-197. doi:10.1016/j.jaccedu.2015.06.001

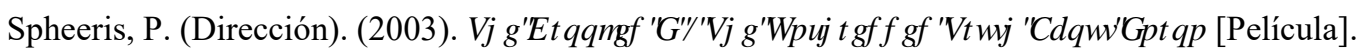
Available at: https://www.youtube.com/watch?v=AiWKPQAWuug\&feature=youtu.be

Stephenson, S. S. (2017). Reflective ethical decision: A model for ethics in accounting education.

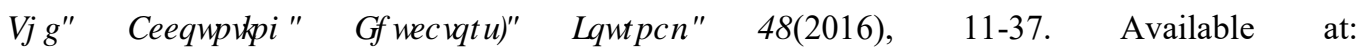
http://www.aejournal.com/ojs/index.php/aej/article/view/345

Uysal, Ö. Ö. (2010). Business Ethics Research with an Accounting Focus: A Bibliometric

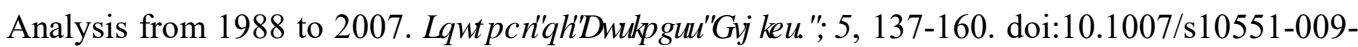
0187-9 